\title{
Mechanical properties of 3D printed composites with ABS/ASA substrate and glass fiber inserts
}

\author{
Cătălin G. Amza ${ }^{1, *}$, Aurelian Zapciu ${ }^{1}$, Arnheiður Eypórsdóttir ${ }^{2}$, Auðbjörg Björnsdóttir ${ }^{2}$, \\ and Jonathan Borg ${ }^{3}$ \\ ${ }^{1}$ University "Politehnica" of Bucharest, Splaiul Independentei 313, sector 6, Bucharest, Romania \\ ${ }^{2}$ University of Akureyri, Akureyri, Iceland \\ ${ }^{3}$ University of Malta, Msida, Malta
}

\begin{abstract}
D printed specimens (ASTM D638 Type I) were manufactured from ABS and ASA material via additive manufacturing through material extrusion 3D printing (ME3DP). During manufacturing, the printing process has been paused, pre-impregnated biaxial or uniaxial glass fiber mesh has been placed onto the ABS/ASA substrate and then the printing process has been resumed. The obtained composite specimens have been subjected to tensile strength testing and results have been compared to those of specimens printed from homogenous material. The influence of raster angle, glass fiber direction and mesh density on resulting toughness has also been analyzed. It was found that inserting uniaxial glass fiber increases toughness of specimens in the axial direction, with a drop in layer adhesion if biaxial fiber is used. Test specimens manufactured with 30 uniaxial fiber strands embedded in a $0^{\circ}$ raster angle 3D printed thermoplastic matrix match mechanical characteristics of injection-molded parts. The maximum mesh density without leading to delamination is one layer of glass fiber every two layers of thermoplastic matrix.
\end{abstract}

\section{Introduction}

Additive Manufacturing (AM) technology, also known as Rapid Prototyping or 3D Printing has been on the market since 1988 when the first machine based on stereolithography was launched. In 2009, a patent for a different AM process, Fused Deposition Modeling has expired which opened the technology to many other users and use cases, as this process enables easy fabrication of three-dimensional parts at a low cost. Nowadays this process is known under many designations such as Fused Filament Fabrication (FFF), 3D Printing or under the standardized name of Material Extrusion 3D Printing (ME3DP).

While ME3DP is cheaper and more user friendly than other fabrication technologies, parts obtained through ME3DP show anisotropic behavior and lower strength than those obtained through other manufacturing techniques [1]. Several methods of increasing the strength and stiffness of 3D-printed parts made from thermoplastic material have been

\footnotetext{
*Corresponding author: acata1@camis.pub.ro
} 
investigated in literature. Some of these methods focus on material science and use carbon, glass or other textile fibers as a filler material included in a thermoplastic matrix, while other methods focus on altering the manufacturing process to include extra steps. This is done either during part fabrication, by inserting fibers in the part while it is being printed or by adding other processing steps after the printing process has completed. Tekinalp et al have analyzed the mechanical properties of parts produced with ABS thermoplastic material filled with highly oriented carbon fibers and found an increase of tensile strength and an almost linear Young's Modulus increase with the content of chopped carbon fibers [2]. Nakagawa et al. have used carbon fiber strands embedded into the feedstock ABS filament and found that strength increases occur after fabrication and microwave treatment [3]. Belter et al. have used infusion of epoxy resin into hollow 3D-printed ABS parts as a post processing technique, leading to increases in part strength and stiffness due to the superior mechanical properties of the thermoset resin [4]. Each of these techniques have their own specific disadvantages and limitations, such as material-related problems (due to abrasion, clogging) in the case of filled thermoplastic build materials, increased system complexity and cost in the case of fiber inclusion during printing or geometry limitations in the case of material infusion post-printing.

\section{Method and materials}

$\mathrm{AM}$ is the process of fusing material to fabricate objects based on data from digital 3D models. Using this process, parts are fabricated by depositing layers of material one on top of the other, unlike non-additive manufacturing processes that use material removal or redistribution to obtain the final part geometry. During ME3DP, thermoplastic material is melt and extruded through a heated nozzle and deposited onto a printing platform following a path which forms one layer at a time. Once a layer is completed the platform or the extrusion head moves by one layer height and starts depositing the next layer of material. This paper presents mechanical testing results of specimens containing glass fiber mesh fabricated by pausing the printing process and placing fiber on top of the last deposited layer. The printing process is then resumed and newly deposited thermoplastic material covers the fiber mesh (fig. 1). Clamps are used to hold the mesh in place until newly deposited material fixes it to the substrate.

Test specimens have been fabricated using two types of commercially available glass fiber mesh: biaxial mesh and uniaxial mesh made from 4-5 parallel fiber strands. Both mesh types have individual fiber strands $0.30 \mathrm{~mm}$ wide and $0.12 \mathrm{~mm}$ thick.

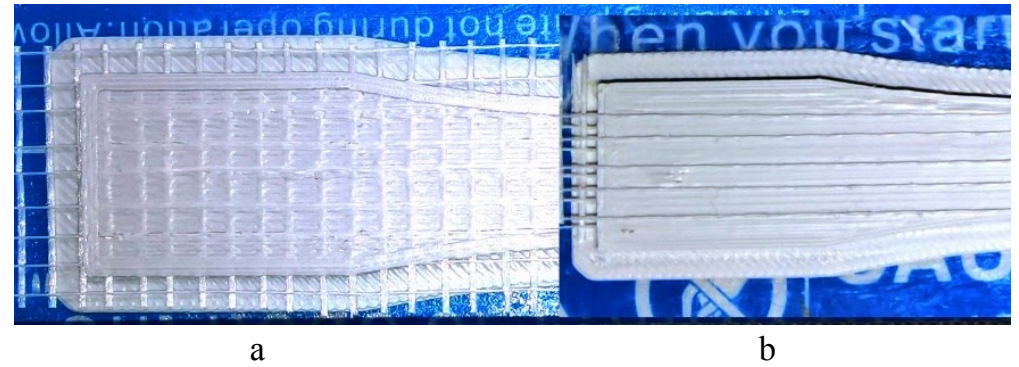

Fig. 1. Glass fiber mesh layering on top of 3D printed substrate layer a) biaxial fiber; b) uniaxial fiber

The materials used as substrate for the glass fibers are acrylonitrile-butadiene-styrene (ABS) and acrylonitrile styrene acrylate (ASA) which are polymers widely used in the consumer goods and automotive industries. These materials are PrimaSelect ABS - White (Prima Filaments, Sweden) and ApolloX - Light Grey (Formfutura, The Netherlands) and 
come as 750 gram spools of $1.75 \mathrm{~mm}$ diameter filament. Test specimens made from the two polymers have been printed with two different raster angle settings, with Type A raster $45^{\circ} / 45^{\circ}$ and Type B raster of $0^{\circ}$ (fig. 2). Part infill has been set at $100 \%$ for the specimens made from homogenous material and at $90 \%$ for the parts with inserted glass fiber. This variance was done to prevent an unwanted buildup of material, as the glass fibers would otherwise occupy the same volume as the thermoplastic material that needs to be deposited. For specimens made with biaxial fiber, 7 layers of fiber mesh have been used, one layer of fiber for each two layers of thermoplastic material, excluding the top and bottom layers. For specimens made with uniaxial fiber, 7 layers of fiber mesh with 4-5 fiber strands each have been used. One layer of glass fiber has been included every two layers of thermoplastic material, excluding the top and bottom layers. The total number of glass fiber strands embedded in a specimen in this case is 30 . The maximum number of mesh layers has been determined through trial and error. Attempting to embed one layer of mesh into every layer of 3D-printed thermoplastic material leads to material buildup and print failure. Additional process parameters used to manufacture the test specimens can be found in Table 1. Model layers and extruder pathing have been created using Simplify 3D v4.1 (Simplify3D, OH, USA). Specimens have been printed with a Qidi Tech IV 3D printer (Ruian Qidi Technology, China) and the same machine command file has been used for both ABS and ASA specimens.

Table 1. Process parameters for printed specimens.

\begin{tabular}{|c|c|c|}
\hline Parameter & Specimen Type A & Specimen Type B \\
\hline Section size & \multicolumn{2}{|c|}{$13.6 \times 3.2 \mathrm{~mm}$} \\
\hline Nozzle diameter & \multicolumn{2}{|c|}{$0.4 \mathrm{~mm}$} \\
\hline Layer height & \multicolumn{2}{|c|}{$0.20 \mathrm{~mm}$} \\
\hline Wall thickness & $1.2 \mathrm{~mm}$ & N/A \\
\hline Printed raster & $-45^{\circ} / 45^{\circ}$ & $0^{\circ}$ \\
\hline Printed infill & \multicolumn{2}{|c|}{$90 \%$ or $100 \%$} \\
\hline Max print speed & \multicolumn{2}{|c|}{$50 \mathrm{~mm} / \mathrm{s}$} \\
\hline Extruder temperature & \multicolumn{2}{|c|}{$240{ }^{\circ} \mathrm{C}$} \\
\hline Platform temperature & \multicolumn{2}{|c|}{$75^{\circ} \mathrm{C}$} \\
\hline
\end{tabular}

All specimens have been fabricated according to ASTM D638 Type I dimensions with a thickness of $3.2 \mathrm{~mm} \mathrm{[5]} \mathrm{and} \mathrm{have} \mathrm{been} \mathrm{subjected} \mathrm{to} \mathrm{tensile} \mathrm{strength} \mathrm{testing} \mathrm{on} \mathrm{an} \mathrm{Instron}$ 8872 machine equipped with an extensometer. Specimens have been fixed in the machine using a hydraulic clamp and have been loaded at a speed of $5 \mathrm{~mm} / \mathrm{min}$ (fig. 3).

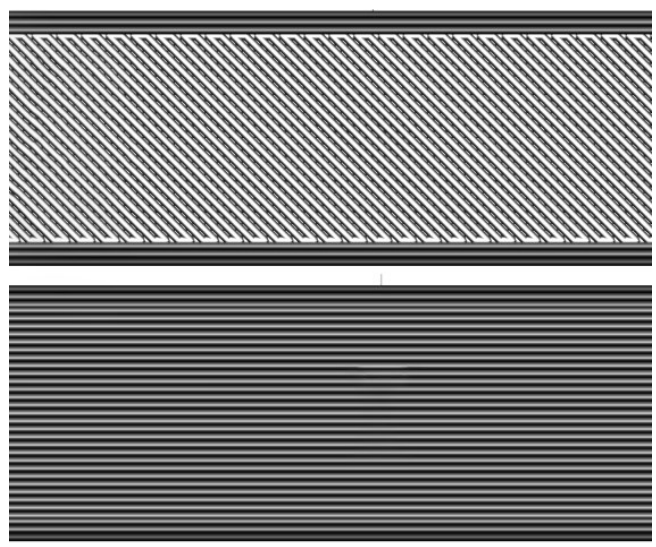

Fig. 2. Raster angle for printed specimens

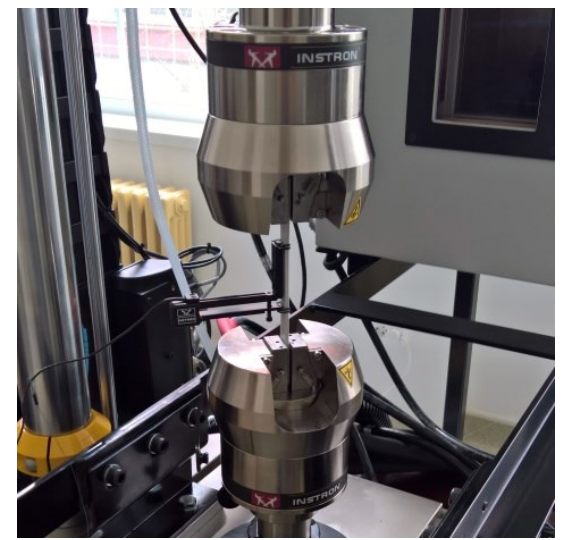

Fig. 3. Tensile strength test setup 


\section{Results and discussion}

For specimens made with homogenous material, the measured tensile strength (fig. 4-6) is on par with other measurements found in literature, with parts made with a $0^{\circ}$ raster angle from ASA material deforming plastically and shearing without fracturing completely even at elongations higher than $15 \%$, while parts with $-45^{\circ} / 45^{\circ}$ raster angle broke under smaller loads, at elongations between $2 \%$ and $3 \%$. As expected, ASA performed slightly better than ABS when printed with identical process parameters. A large dip in performance was observed for $\mathrm{ABS}$ specimens printed with $-45^{\circ} / 45^{\circ}$ raster angle and we suspect this is due to inadequate material bonding between deposited filament roads due to temperature conditions (print temperature $240{ }^{\circ} \mathrm{C}$, platform temperature $75{ }^{\circ} \mathrm{C}$ ). It can also be observed that generally, mechanical behavior transition from elastic to plastic deformation happened at a higher load in the case of $0^{\circ}$ raster angle parts. Inserting biaxial glass fiber mesh had no noticeable positive effect in the case of $45^{\circ}$ raster parts. In many of these specimens, the thermoplastic matrix fractured before the glass fibers were fully loaded and the presence of transversal glass fibers had a weakening effect on the parts.

The best results are achieved in the case of parts printed with $0^{\circ}$ raster angle filled with uniaxial glass fiber. Tensile strength of ASA composite was $16 \%$ higher compared to homogenous material after the inclusion of 7 layers of uniaxial fiber (30 fiber strands in total). Figure 7 shows the fracture modes for selected specimens. For parts printed with a $45^{\circ}$ raster angle, fracture occurs along filament roads, at $45^{\circ}$ angles. The different modes of failure for $0^{\circ}$ raster parts made from homogenous $\mathrm{ABS}$ and ASA also highlight their difference in elasticity.

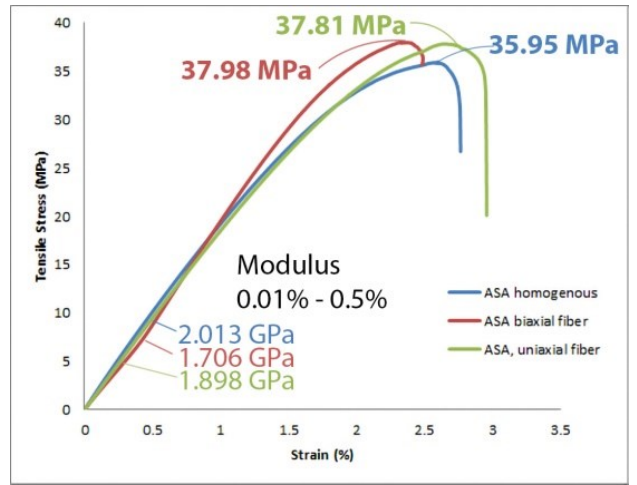

Fig. 4. Tensile strength testing of ASA specimens with $-45^{\circ} / 45^{\circ}$ raster angle



Fig. 6. Tensile strength testing of ABS specimens

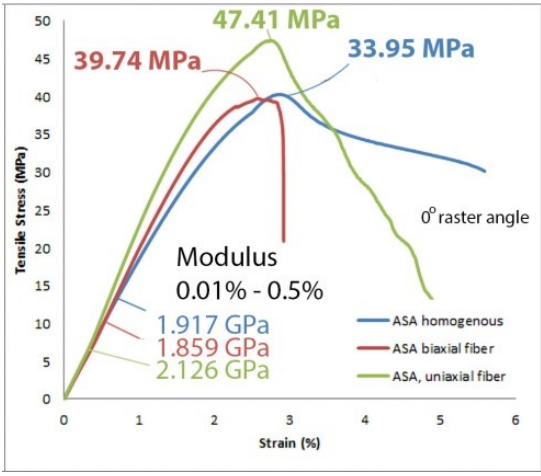

Fig. 5. Tensile strength testing of ASA specimens with $0^{\circ}$ raster angle

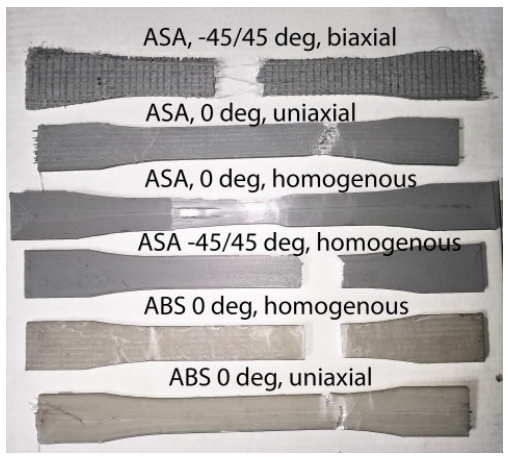

Fig. 7. Failure modes for selected specimens 
Some observations can be made regarding the defects that can appear during specimen fabrication and their influence on the mechanical properties of the resulting part. During the printing process, because the location of the glass fiber strands is unknown to the 3D printing machine, when the extrusion head passes transversely over a strand of fiber a small buildup of material occurs on one side of the strand, and an empty gap in the part forms on the other side of it (fig. 8a). This results in the creation of a weak point in the part. With the insertion of biaxial glass fiber mesh that has strands perpendicular to the direction of the load, these weak points are aligned and act as a catalyst for part fracture. In future iterations of the fiber insertion process, this type of defect could be corrected with methods such as computer vision to identify the location of the glass fiber strands and adjust the material extrusion flow accordingly.

For some of the specimens manufactured with a $-45^{\circ} / 45^{\circ}$ raster angle, total fractures occurred before the embedded glass fibers were fully ripped. As a result, the specimen could fracture with many of the fiber strands still intact (fig. 8b). We suspect this happens when the glass fiber strands are stressed insufficiently because of lack of pretensioning when laying the mesh, as Young's Modulus for the glass fiber strands is significantly higher than that of the ABS/ASA thermoplastic matrix.



a

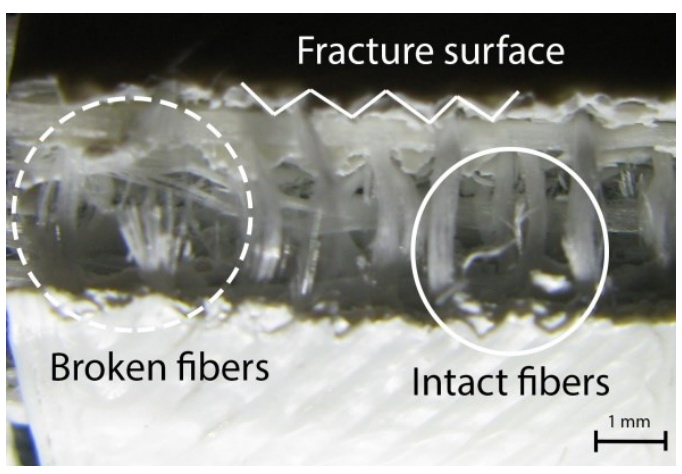

b

Fig. 8. Defects causing premature specimen rupture a) Void formation with transverse extrusion direction; b) Intact fibers due to lack of fiber tension

\section{Conclusions}

This paper focused on fabricating and testing 3D printed specimens with insertions of fiberglass mesh.

A series of specimens have been fabricated through ME3DP from ABS and ASA material. During the printing process, one of two types of glass fiber has been inserted between the printed layers in order to increase material strength. Tensile tests done on the printed specimens show an increase in tensile strength for parts printed with a $0^{\circ}$ raster angle and a slight decrease in strength for parts printed with a $-45^{\circ} / 45^{\circ}$ raster angle when using biaxial glass fiber mesh. This is due to defects forming at the intersection of extruder trajectory and mesh strands and to weakening of thermoplastic matrix caused by the presence of transversal fiber strands. The maximum mesh density achieved is one layer of glass fiber mesh every two layers of printed material.

Biaxial glass fibers had no significant positive effect on parts printed with a $-45^{\circ} / 45^{\circ}$ raster angle.

Uniaxial glass fibers increase material strength by $5-8 \%$ for parts printed with a $-45^{\circ} / 45^{\circ}$ raster angle and by $12-16 \%$ for parts printed with a $0^{\circ}$ raster angle, when loads are applied in the direction of the fiber strands. It has been observed that pretensioning the fibers leads 
to better results (higher tensile strength), as the material can break before fibers are fully tensioned if pretensioning is not used.

Composites made with uniaxial glass fiber (30 fiber strands in total) in an ASA or ABS matrix printed at $0^{\circ}$ raster angle exhibited tensile strength of $47.4 \mathrm{MPa}$ and $44.2 \mathrm{MPa}$ respectively, matching or exceeding experimentally determined tensile strength of homogenous material injection molded parts found in literature [6].

A potential direction for future work could address the possibility of fully or partially automating the fiber insertion process in order to shorten manufacturing times and mitigate resulting defects. Other directions are testing other combinations of fibers and matrix materials, as well as looking into changes resulting from using different process parameters.

This result has been financed through the EEA cooperation project no. EY-COP-0001, funded by EEA Grants 2014-2021.

\section{References}

1. SH. Ahn, M. Montero, D. Odell, S. Roundy, P.K. Wright, Rapid Prototyp J., 8(4), 248 - 257 (2002)

2. H. L. Tekinalp, V. Kunc, G. Velez-Garcia, C.E. Duty, L. Love, A.K. Naskara, C. A. Blueb, S. Ozcan, Compos Sci Technol. 105, 144-150 (2014)

3. Y. Nakagawa, K. Mori, T. Maeno, Int J Adv Manuf Tech. 91(5-8), 2811-2817 (2017)

4. JT Belter, AM. Dollar, PLoS ONE 10(4), e0122915 (2015)

5. ASTM D638 - 14 Standard Test Method for Tensile Properties of Plastics, https://www.astm.org/Standards/D638 last accessed 20 February 2019

6. D. Palmer, Plast Technol. 60(10), 56-58 (2014) 